\title{
Guest editorial: Focused section on smart mobile robotic systems in challenging environments
}

\author{
Shaohui Foong ${ }^{1} \cdot$ Xianbo Xiang $^{2}$ \\ Published online: 3 March 2020 \\ (c) Springer Nature Singapore Pte Ltd. 2020
}

Robots, unlike humans, are designed and engineered to operate in a variety of extreme conditions. In many applications where human presence is dangerous, difficult or undesirable, these mobile robotic systems remain the only method of performing and completing critical tasks in the air, on land and above water or underwater. In order to operate efficiently and demonstrate high intelligence and autonomy, these mobile robotic systems would also need to incorporate intelligent sensing, perception, actuation, control and design synthesis.

The International Journal of Intelligent Robotics and Applications (IJIRA) has previously published a "Focused section on smart mobile robotic systems in challenging environments" in June 2019 to document and disseminate current advances, challenges and opportunities in this domain. Immense growing interest and demand in this emerging area has stimulated a second edition of this focused section. This follow-up edition of the focused section highlights recent efforts and significant results in smart mobile robotic systems designed and developed for the challenging aerial and ground environments. A total of five papers have been selected for this focused section that represent the current ongoing developments of intelligent mobile robotic platforms operating in challenging environments.

The aerial domain has always been a fascinating environment and three of the five papers focus on analysis and development of smart mobile robots that fly. The first paper "Energetic Analysis and Optimization of a Bi-Modal

Xianbo Xiang

xbxiang@hust.edu.cn

Shaohui Foong

foongshaohui@sutd.edu.sg

1 Engineering Product Development (EPD) Pillar, Singapore University of Technology and Design (SUTD), 8 Somapah Road, Singapore 487372, Singapore

2 School of Naval Architecture and Ocean Engineering, Huazhong University of Science and Technology, Luoyu Road 1037, 430074 Wuhan, People's Republic of China
Rolling-Flying Vehicle" from Gregory D. Buckner, presents a theoretical energy orientated analysis of a novel bi-modal unmanned aerial vehicle (UAV) that is capable of rolling and flying. This rolling-flying vehicle (RFV) combines the mobility and maneuverability of rotary wing flight with the efficiency of rolling locomotion. Using blade element momentum theory and an electromechanical motor model, detailed simulations were used to investigate the energy consumption as well as operational range of the RFV and when compared to a conventional multirotor, it was found that the RFV was around ten times as efficient. The paper also found that both the angle of attack and velocity of the RFV play critical roles in determining the optimal flying configuration of the RFV.

The second aerial-based paper by Abouheaf and Gueaieb on "Online model-free controller for flexible wing aircraft: a policy iteration-based reinforcement learning approach" employs machine learning techniques to design a robust online model-free control scheme for flexible wing aircraft. Hang gliders are an example of flexible wing aircraft and this class of aircraft offers many advantages over conventional fixed-wing UAVs. The main challenge is that the aerodynamic model of flexible wing aircrafts is highly nonlinear with continuously time-varying dynamics under kinematic constraints. Developing controllers based on these nonlinear models which stem from the continuous deformations in the flexible wings are especially difficult. The adaptive learning controller uses a policy iteration approach and artificial neural networks to implement the adaptive learning algorithm in real-time. In addition to be model-free, the proposed controller is also robust to the uncertainties in the system's highly coupled dynamics.

The third and final aerial robotic paper is on "A Development Platform for Behavioral Flexibility in Autonomous Unmanned Aerial Systems" by Bodin et al. Motivated by the potential of autonomous UAVs to subsume a substantial portion of missions currently carried out by remote pilots, this paper presents a development UAV platform for ease of 
deploying and evaluating new autonomous framework and capabilities. This platform proposed by the authors incorporates a Unified Behavior Framework (UBF) operating on the open source Robotic Operating System (ROS) with the PX4 open source flight controller in tandem. The development platform presented in this work offers robust and responsive behavioral flexibility for UAS agents in simulation and reality using a methodology originally proven on ground robots.

Simultaneous localization and mapping (SLAM) of an unknown environment is a critical requirement for any autonomous robot operating in challenging environments. The fourth paper presents a cost effective, robust and integrated software/hardware SLAM solution for 2D mapping of large indoor environments. This paper "MapperBot/iSCAN: Open-Source Integrated Robotic Platform and Algorithm for 2D Mapping" from Al-Hourani and Ristic introduces a SLAM algorithm, called iSCAN, which is based on a highly robust laser-scan matching technique, incorporating RaoBlackwellized particle filter as a means to increase its accuracy. As a result, a minimal number of particles is required, and the computer processing time is dramatically reduced. Paired with the SLAM algorithm is the MapperBot hardware that is built using commercial of-the-shelf components with an open-source software/hardware design. The composite MapperBot/iSCAN solution is fully integrated with the MATLAB environment, thus providing researchers, students and developers a quick entry into the realm of the SLAM.

Last but not least, the final paper addresses the need to develop affordable and accessible platforms for students to explore and assess deep learning visual based algorithms for mobile robots. The paper "An educational Arduino robot for visual Deep Learning experiments" by $\mathrm{He} \mathrm{Hu}$ acknowledges the role played by mobile robots as a vital education tool for students and novices. Many educational institutions in the world, especially those in developing countries, cannot afford expensive robots which can support deep learning experiments. As mobile phones are relatively inexpensive and significantly more accessible, the proposed mobile robot in the paper uses an attached android mobile phone to capture images and video streams as well as having the capability of running visual deep leaning models through a direct wireless link to a connected computer.

We would like to express our sincere thanks to all of the authors and anonymous reviewers as well as the production colleagues for their tireless efforts and dedicated contributions to this Focused Section. Our appreciation also goes to Editor-in-Chief, Professor Kok-Meng Lee, and editors and for their wisdom and hard work in coordinating the timely and vigorous review of all submitted papers.
Publisher's Note Springer Nature remains neutral with regard to jurisdictional claims in published maps and institutional affiliations.

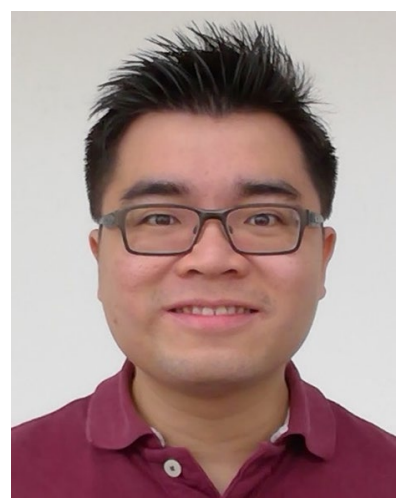

Shaohui Foong is an Associate Professor in the Engineering Product Development (EPD) pillar at the Singapore University of Technology and Design (SUTD) and Visiting Academician at the Changi General Hospital, Singapore. He received his B.S., M.S. and $\mathrm{Ph} . \mathrm{D}$. degrees in Mechanical Engineering from the George W. Woodruff School of Mechanical Engineering, Georgia Institute of Technology, Atlanta, USA in 2005, 2008 and 2010. In 2011, he was a Visiting Assistant Professor at the Massachusetts Institute of Technology, Cambridge, USA. He has a keen interest in mechatronics, which is the synergistic integration of the multiple disciplines of mechanics, electronics, control theory and computer science, and much of his research is centred around designing and developing novel mechatronic systems for a wide spectrum of applications. His research interests include system dynamics \& control, natureinspired robotics, magnetic localization, medical devices and design education \& pedagogy.

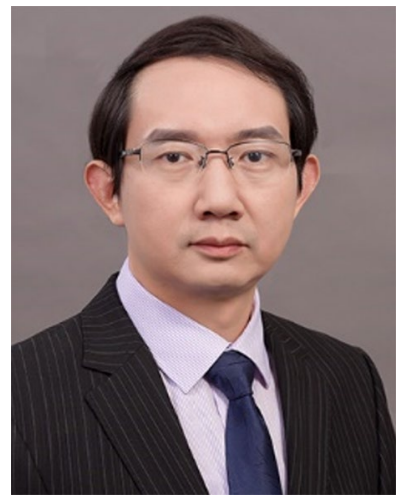

Xianbo Xiang is a Professor in the School of Naval Architecture and Ocean Engineering, Huazhong University of Science and Technology. He received the B.E. and M.E. degrees in automatic control and marine engineering from the Huazhong University of Science and Technology, China, in 2000 and 2003, respectively, and the Ph.D. degree in System Automation and Micro-electronics from the University of Montpellier II, France, in 2011. In 2006, he was an EU Erasmus Mundus Visiting Scholar in the SpaceMaster Project for three months. From 2008 to 2011, he was in the European Project FreeSubNet as an EC Marie Curie ESR Fellow with the LIRMM, CNRS UMR 5506, Montpellier, France. He has served as an International Editorial Scientific Advisory Board Member of Journal Brodogradnja/Shipbuilding, and as the guest editors for International Journal of Fuzzy Systems and Indian Journal of Geomarine science. He was the general chair of the 2018 IEEE 8th International conference on Underwater System Technology: Theory and Applications (USYS'18). His current research interests include robotics and marine vehicle systems. 